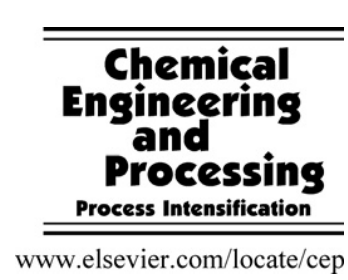

\title{
The use of LDS as a tool to evaluate flocculation mechanisms
}

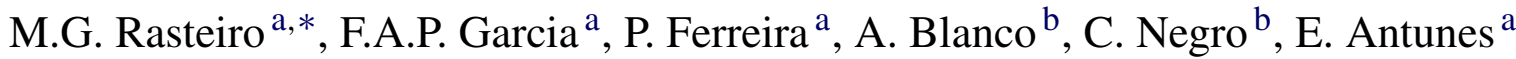 \\ ${ }^{a}$ Chemical Engineering Department, Coimbra University, Pólo II, Pinhal de Marrocos, 3030-290 Coimbra, Portugal \\ ${ }^{\mathrm{b}}$ Chemical Engineering Department, Faculty of Chemistry, Complutense University, 28040 Madrid, Spain
}

Received 4 August 2006; received in revised form 2 April 2007; accepted 27 April 2007

\begin{abstract}
Flocculation studies of precipitated calcium carbonate induced by cationic polyacrylamides (C-PAMs) were carried out using light diffraction scattering (LDS). The effect of both polymer charge density and concentration on the flocculation process and on flocs density was investigated. As expected, results show that high charge density C-PAM induces flocculation by bridging and patching mechanisms simultaneously, while medium charge density C-PAM acts mainly according to the bridging mechanism. Consequently, the mass fractal dimensions of the flocs produced by high charge density C-PAM are higher. Results also show the effect of flocculant concentration: flocculation rate decreases and denser flocs are obtained as flocculant concentration increases. The results obtained so far allowed a preliminary quantitative evaluation of flocculation kinetics. In the flocculation curve, two regions corresponding to different kinetics were identified: a first region dominated by particle aggregation and a second region dominated by flocs stabilization. Therefore, LDS is considered a useful tool to evaluate flocculants performance. A strategy was developed that resulted in the use of LDS to retrieve, in a single test, information on the evolution with time of flocs dimension and structure, flocs resistance and flocculation kinetics. All the tests were performed under turbulent conditions similar to the ones prevailing in process equipment. (C) 2007 Elsevier B.V. All rights reserved.
\end{abstract}

Keywords: Flocculation mechanisms; Laser diffraction spectroscopy; LDS; Charge density; Mass fractal dimension; Floc structure

\section{Introduction}

Aggregation of particles is fundamental for improving the performance of many solid/liquid separation processes. Aggregation can be induced by different means one of the most common being based on the addition of polymeric additives, namely polyelectrolytes. Flocculation by polyelectrolytes is of importance to many industrial areas, namely in food manufacturing, pharmaceuticals and also in papermaking, and is well documented in the literature [1-6].

In the case of the papermaking process, flocculants are used as retention or drainage aids and, therefore, flocculation plays an important role on runnability, product quality and economy of the process. Thus, it is important to know, for each flocculant, the predominant interactions and mechanisms of fines and filler retention, in order to optimize the corresponding flocculation process [7]. Though the strategy developed with the present

\footnotetext{
* Corresponding author. Tel.: +351 239798700; fax: +351 239798703 .

E-mail address: mgr@eq.uc.pt (M.G. Rasteiro).
}

study is not restricted to a specific application, it is mainly directed to flocculation in papermaking.

Various processes occur simultaneously during flocculation: adsorption of polymer molecules at the particles surface; rearrangement (or re-conformation) of adsorbed polymeric chains; collisions between destabilized particles to form aggregates (flocs); and break-up of flocs $[1,2,4]$. The importance of each process depends on the flocculant characteristics, like structure, molecular weight, charge density and concentration; on the characteristics of the suspended particles, like size and charge density; on the characteristics of the suspending medium, like $\mathrm{pH}$, conductivity and ionic charge; and, finally, on the contact time and turbulence intensity, among others $[2,3,8,9]$.

One of the aforementioned parameters that most affects flocculation is the charge density of the polyelectrolyte, because it determines its conformation when adsorbed on the particle surface, and, therefore, the predominant flocculation mechanism $[9,10]$. In general, if molecular weight is high and charge density is low, the polymer adsorbs on the particle surface in such a way that tails and loops are extended far beyond the surface and can interact with other particles - in this case the flocculation process is dominated by bridging bonds $[4,8]$. When the charge 
density is high, the bridging capability is reduced because there is a tendency for the polymer chains to adopt a flatter conformation on the particle surface, which results in the formation of cationic patches that attract the polymer free surfaces of other particles $[8,11]$. In this case, the adsorption rate becomes lower and the conformation rate becomes faster as the cationic charge of the polymer increases.

The concentration of the flocculant is also a key parameter, since the rate of adsorption depends on the amount of polymer adsorbed per unit area of the particle surface. Tadros [12] proposed the "diffusion-controlled adsorption kinetics model," stating that the flux of adsorption dominates when the surface concentration of polymer is lower than the equilibrium concentration, whereas desorption is the ruling phenomena when the surface concentration is higher than the equilibrium concentration. Moreover, the flocculant concentration also affects the conformation rate: polymers re-arrange relatively fast at low surface concentration but rather slowly on crowded surfaces, since neighbouring molecules interfere with the re-arrangement $[4,13]$. In addition, for flocculation to occur, polymer molecules have to collide with the fine particles in order to be adsorbed, and the polymer-coated particles have also to collide with each other [1]. Thus, mixing is essential to promote flocculation. However, as flocs become larger, further growth is restricted by the applied shear that erodes or breaks down the flocs depending on their size [14]. In fact, the hydrodynamic forces that tend to disrupt the flocs become greater as their size increases and the collision efficiency of the particles becomes lower. Therefore, there is a limiting size for flocs growth, determined by the balance between aggregation and breakage $[1,2,15,16]$.

Despite the enormous amount of information already existing on flocculation processes promoted by polymeric additives, this is still an area of continuous debate and research. On one hand, there are still no general models that allow the prediction of flocculation characteristics, namely floc properties, as a function of all the different parameters that influence flocculation. On the other hand, it is not yet available a standard experimental strategy, widely accepted, that could be used to test the new flocculants that are continuously being developed, as will be discussed in the next paragraphs. This is commonly admitted in the reports of researchers active in this field, even in recent publications $[4,5]$.

Traditional methods for optimizing the polymer dosage are based on the DVLO theory, which relates the optimum dosage to the zero zeta potential $(\zeta)$ [12]. This theory is valid when flocculation occurs by charge neutralization, but does not fit when medium or high molecular weight polymers are used and the bridging or patching mechanisms dominate $[7,9,17,18]$. Consequently, the methods based on electrokinetic parameters or polyelectrolytes titration should be used with great caution. Nevertheless, zeta potential has been used to monitor flocculation, the decrease in this parameter corresponding to a decay of the surface charge during the flocculation process (as a result of the transition from the bridging to the charge neutralization mechanism) [19,20].

Another traditional technique to monitor flocculation and to assess the performance of flocculants is based on settling tests in the absence of turbulence, monitored by different means $[5,6]$, which can supply indirect information on flocs average size and structure [21] (namely the mass fractal dimension) for the conditions prevailing in a sedimentation system.

As an alternative, Blanco et al. [7,8] developed a method for optimizing the polymer dosage based on monitoring the growth of the particle aggregates during flocculation: the mean floc size increases when polymer is added, but when the total added dosage is higher than the optimal one no more aggregation takes place and the mean size can even decrease due to steric stabilization and/or electrostatic repulsion. To measure the particle size of the aggregates, a focused beam reflectance-measuring probe (FBRM) was used to get information about the average chord of the aggregates and the number of counts.

On the other hand, recent studies have shown that light diffraction scattering (LDS) is a useful technique to monitor the dynamics of flocculation and to evaluate the influence of the flocculant characteristics and dosage [22]. LDS not only allows the determination of the aggregate mean size and size distribution, but gives also the mass fractal dimension of the flocs, $d_{\mathrm{F}}[4,21,23]$. The mass fractal dimension provides a mean of expressing the degree to which primary particles fill the space within the nominal volume occupied by an aggregate and is, therefore, a convenient parameter to characterize the density of the flocs [24]. For solid non-porous particles $d_{\mathrm{F}}=3$ and for porous particles $1<d_{\mathrm{F}}<3[25]$.

It is well reported in the literature that floc strength depends on floc structure and density. Tang found that the mechanical strength of the floc depends on both the particle forces and on how the particles are packed within the aggregate [24]. The stronger the bonding force between the particles, the higher the floc strength. Similarly, the more compact the floc structure, the higher the number of interparticle bonds resulting in stronger flocs. Therefore, the prediction of floc strength as a function of flocculant type and operating conditions is very important for industrial applications. LDS allows also an evaluation of flocs resistance at the end of the flocculation process, for different shear rates. The tests can be programmed so that the dynamics of aggregation, the resistance of the fully stabilized flocs and their reflocculation ability can altogether be evaluated in a single experiment.

In this study, after a preliminary analysis of the techniques available to follow flocculation processes, it was decided to select the LDS technique to monitor flocculation. In fact, the results obtained prove that LDS possesses the capability to allow an integrated evaluation of flocculants performance, by supplying, simultaneously, information on flocs size distribution, average size and mass fractal dimension, in a continuous way, as time elapses, if the equipment is pre-programmed for continuous data acquisition. Moreover, flocculation can be processed in the equipment dispersion unit in controlled turbulent conditions that can simulate more easily what is happening in the process itself.

Results obtained for assessing the flocculation mechanism of two different high molecular weight cationic polyacrylamides (C-PAMs) are presented and discussed in this paper. The effects of the flocculant charge density and concentration on the flocs 
size and structure and on the flocculation process, including flocculation kinetics and mechanism, were investigated. Because of the importance of filler retention for papermakers the flocculation of precipitated calcium carbonate (commonly used as filler in papermaking), under turbulent conditions, has been studied. With this study, the capacity and added value of the LDS technique to evaluate in an integrated way (single programmed test) the size, structure and strength of the flocs, as well as the flocculation mechanism and kinetics, are clearly demonstrated.

\section{Experimental}

\subsection{Materials}

An aqueous suspension of a commercial PCC, at 1\% (w/w), produced in a satellite unit of a paper mill, was used throughout this work. In order to obtain a preliminary good dispersion of the particles, the suspension was submitted to magnetic stirring at $600 \mathrm{rpm}$ during $40 \mathrm{~min}$ and then to sonication at $50 \mathrm{kHz}$ during $15 \mathrm{~min}$. After this treatment, the median size of the particles was approximately $0.5 \mu \mathrm{m}$ and the suspension $\mathrm{pH} 7.5$.

Two new C-PAM emulsions of high molecular weight, developed and supplied by AQUA + TECH, were used in this study: Alpine-Floc ${ }^{\mathrm{TM}}$ BHMW, a linear polyacrylamide with a molecular weight around $7.2 \times 10^{6} \mathrm{~g} / \mathrm{mol}$ and $80 \%$ (w/w) of groups charged, and Alpine-Floc ${ }^{\mathrm{TM}} \mathrm{E} 1+$, a low branched polyacrylamide with a molecular weight of $13 \times 10^{6} \mathrm{~g} / \mathrm{mol}$ and $50 \%$ (w/w) of groups charged. The cationic monomer of both polymers is dimethylamino ethyl acrylate. For the experiments, BHMW was diluted to $0.05 \%$ and $\mathrm{E} 1+$ to $0.1 \%$ in distilled water. In order to guarantee the effectiveness of the flocculants, the diluted solutions were prepared everyday and the distilled water conductivity was controlled between 5 and $8 \mu \mathrm{S} / \mathrm{cm}$, since significant variations of this parameter strongly affect the flocculation process.

\subsection{Methods}

The flocculation of the PCC particles was monitored by measuring the size of the aggregates by LDS in a Malvern Masterziser 2000 equipment (Malvern Instruments). The LDS technique is based on the analysis of the light scattered by the particles when they interfere with a coherent light beam, in the present case a $\mathrm{He} / \mathrm{Ne}$ laser beam. If the scattered light can be related mainly to the diffraction mechanism, then the angle of diffraction is associated with the size of the particles, larger particles diffracting light for smaller angles and smaller particles for larger angles [26]. In the Malvern Mastersizer 2000 the scattered light is collected, in the forward direction, by 52 concentric photodiodes. The scattering patterns obtained are processed using an appropriate optical model in order to calculate the particle size distribution. Traditionally, two different models are used: the Fraunhofer approximation and the Mie theory [27]. In this study, we use the Mie theory, since it provides a more rigorous solution for the calculation of particle size distribution from light scattering data, mainly when particles smaller than $10 \mu \mathrm{m}$ are considered. The scattering data allows also one to calculate the mass fractal dimension from the negative slope of the $\log -\log$ plot of the scattered light intensity as a function of the wavenumber [28].

The $1 \%$ (w/w) PCC suspension was added to $700 \mathrm{~mL}$ of distilled water until $30 \%$ obscuration of the laser beam was reached (total solids concentration around $0.02 \%$, w/w), and the tests were carried out by setting the pump speed to $1400 \mathrm{rpm}$. Obscuration is a good control parameter to evaluate the signal quality and, according to the manufacturers, it must be kept, ideally, between 5 and 20\%. However, as obscuration decreases during the measurements, due to the reduction of the number of scattering entities, the initial obscuration was set to $30 \%$ so that, at the end of the flocculation test, obscuration was always above $5 \%$ in order to guarantee the validity of the results [22]. Previous studies have shown that the tests could be conducted in a dilute state in the equipment dispersion unit, since similar results were obtained when flocculation was conducted at a higher concentration in an outside beaker and a sample was then rapidly transferred to the dispersion unit of the LDS equipment and analysed [22]. The reported values of the median particle size $\left(d_{\mathrm{p} 50}\right)$ represent an average of at least four replications and, in each case, the measured values lied within a range that was less than $\pm 3 \mu \mathrm{m}$ around the central value; for the reported highest median sizes $\left(d_{\mathrm{p} 50}>80 \mu \mathrm{m}\right)$, the interval of variation was slightly larger but still below $\pm 5 \mu \mathrm{m}$.

Both flocculants were tested with different concentrations, close to the optimum dosage. This optimum was determined following the methodology developed by Blanco [7] but using LDS instead of FBRM to monitor the aggregates size. For that, the flocculant stock solution was previously diluted again to $0.01 \%$ and $1 \mathrm{~mL}$ of the resulting working solution was added to the PCC suspension every minute, until the floc size began to decrease.

The size of the PCC particles was measured before the addition of the flocculant to the suspension. When the tests were designed to monitor the flocculation evolution for a fixed concentration of flocculant, this was added to the PCC suspension at once in the equipment dispersion beaker, and the flocs size was measured every minute during $14 \mathrm{~min}$ (after $14 \mathrm{~min}$ the flocs size seemed to be stabilized). The mass fractal dimension of the flocs during the flocculation process was also computed from the scattering pattern used to determine the particle size, with the objective of evaluating the compactness of the flocs. The individual particles could be considered to follow the Rayleigh-Gans-Debye approximation [21] (particles smaller than $1.0 \mu \mathrm{m}$ and refractive index 1.572).

In addition, the zeta potential of the suspensions was also measured using the Zetasizer NanoZS equipment (Malvern Instruments), at three moments: 1 min after the addition of the flocculant, $7 \mathrm{~min}$ after the addition of the flocculant and at the end of flocculation (14 min).

Optical microscopy with image analysis (Olympus BH-2 microscope with analysSIS 2.11) was used to visualize the flocs shape, at the end of the flocculation process, in order to validate the results obtained by LDS. Only tests for the flocculant concentration optimised by LDS were performed. 


\section{Results and discussion}

\subsection{Optimum flocculant dosage tests}

Fig. 1 shows, for both flocculants, the $d_{\mathrm{p} 10}$ and the median $\left(d_{\mathrm{p} 50}\right)$ equivalent spherical diameters of the flocs as a function of the amount of polymer added to the PCC suspension (expressed in terms of $\mathrm{mg}$ of polymer/g of PCC). These tests enabled the selection of a range of flocculant concentrations for the subsequent experiments.

Data show a complete different behaviour of the two CPAMs. In the case of E1+ the optimum dosage was obtained for approximately $9 \mathrm{mg} / \mathrm{g}$, as can be concluded from the evolution of both the $d_{\mathrm{p} 10}$ and $d_{\mathrm{p} 50}$ diameters, which show a clear maximum. For BHMW, these sharp maximums are not observed and the decrease of $d_{\mathrm{p} 50}$ is not so pronounced, but since $d_{\mathrm{p} 10}$ starts to decrease at approximately $10 \mathrm{mg} / \mathrm{g}$ we have considered that this was the optimum dosage for this flocculant. Moreover, the flocs size at the optimum dosage is considerably larger with E1+ than with BHMW.

Considering that E1+ is a C-PAM with a high molecular weight and medium charge density, the aggregation process takes place mainly by the bridging mechanism, as confirmed by the fast flocculation rate (Fig. 2). Concerning BHMW, which has a higher charge density, there is a tendency for the polymer chains to adopt a flatter configuration on the particle surface that produces a reduction of the bridging capability in favour of patching, thus aggregation occurs by bridging and patching simultaneously. As a consequence, the bonds between the particles are shorter, the flocs are smaller and the flocculation rate is in general slower (Fig. 3) [12]. Nonetheless, for low concentrations, the flocs obtained with BHMW are larger than those obtained with E1+. When we start adding the polymer aggregation with BHMW takes place more rapidly than with E1+ since, due to the high charge density, the adsorption rate is higher [29]. After this rapid aggregation, because of the flatter conformation of the polymer on the particle surface, the adsorption of further flocculant becomes slow [29] and flocs size increases very slowly. For E1+, the flocs size decreases abruptly after the maximum that defines the optimum dosage, as consequence of

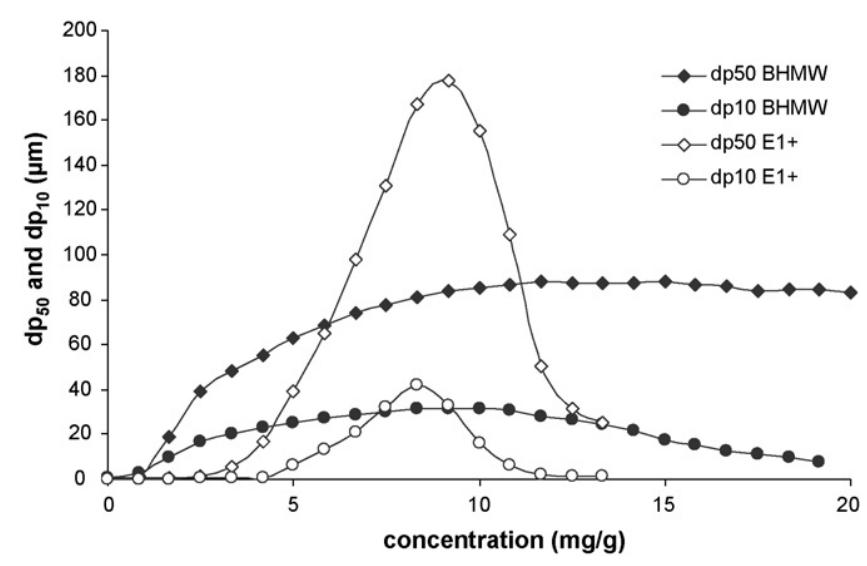

Fig. 1. Optimum flocculant dosage for BHMW and E1+.

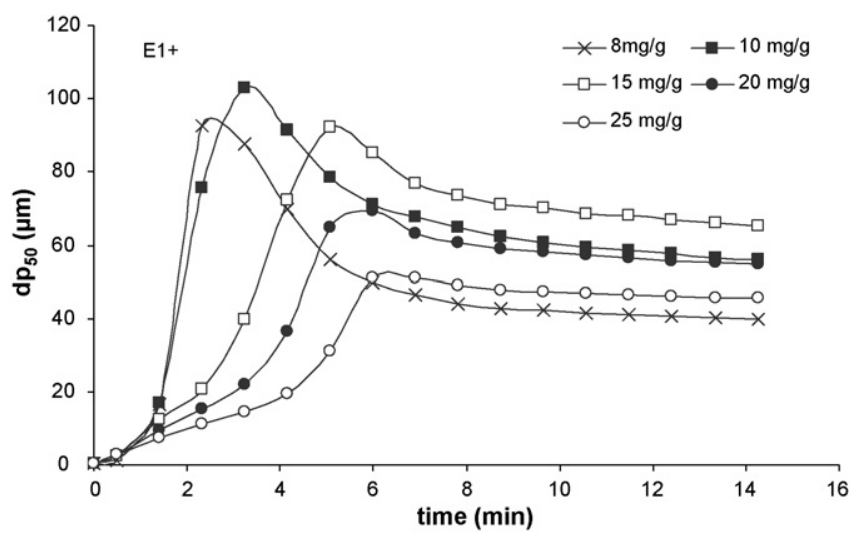

Fig. 2. Median flocs size as a function of flocculation time for several E1+ concentrations

steric and electrostatic repulsion. For BHMW this decrease is not observed. This explanation is in agreement with the values of the mass fractal dimension of the flocs at the point of optimal dosage. In fact, for that point, $d_{\mathrm{F}}=1.74$ and $d_{\mathrm{F}}=1.66$ for BHMW and $\mathrm{E} 1+$, respectively, confirming that the flocs produced with BHMW are more compact.

\subsection{Monitoring of the flocculation process}

For monitoring the flocculation process, the values of the optimum flocculant dosage were used in order to define the adequate range of flocculant concentration: the new tests were performed with 2, 6, 10 and $14 \mathrm{mg} / \mathrm{g}$ of BHMW and with 10, 15, 20 and $25 \mathrm{mg} / \mathrm{g}$ of E1+ but, contrary to the preliminary trials, the flocculant was added to the suspension all at once, in the equipment dispersion beaker.

Figs. 2 and 3 illustrate the flocculation process carried out with E1+ and BHMW, respectively, in terms of the evolution of the floc median size with time as a function of polymer concentration. As it was expected, flocculation rate decreases as the flocculant concentration increases. Since the stirring speed is the same for all the tests, this decrease can be explained by the diffusion-controlled adsorption kinetics model, in such a way that the higher the amount of polymer, the lower the poly-

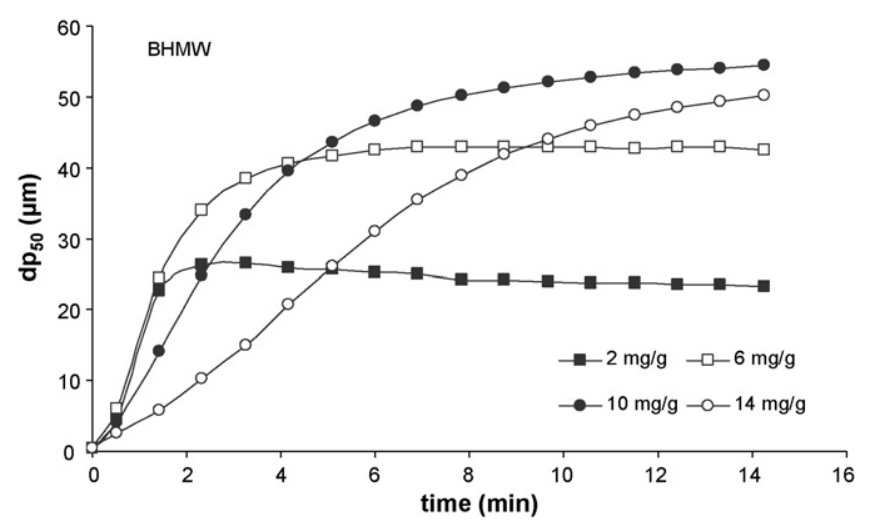

Fig. 3. Median flocs size as a function of flocculation time for several BHMW concentrations. 


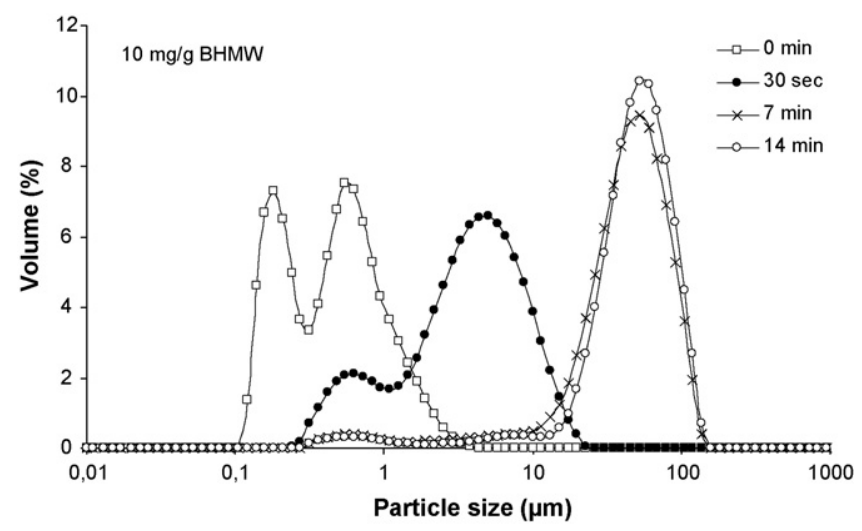

Fig. 4. Flocs size distribution evolution during flocculation process with $10 \mathrm{mg} / \mathrm{g}$ BHMW.

mer adsorption rate on the particle surface [12]. Therefore, the equilibrium point is reached more rapidly when the flocculant concentration is lower and, as a consequence, the final flocs size is reached earlier.

However, at high dosages the optimum coverage rate is higher than the optimum, flocculation is slower and floc size increases very slowly. For very low concentrations of BHMW $(2 \mathrm{mg} / \mathrm{g})$ the flocs size reaches rapidly a maximum and then starts to decrease. This decrease must be due to the fact that, with the rapid flocculation at the beginning, the polymer has no time to adopt a flat conformation. Only after reaching the equilibrium the polymer chains start to reorganize themselves, resulting in flocs compaction, and thus size reduction. For BHMW it may also be concluded that the formation of patching bonds increases with the flocculant concentration and, thus, a better organization of the polymer chains occurs at the beginning. Furthermore, Fig. 4, which refers to a concentration of $10 \mathrm{mg} / \mathrm{g}$ of BHMW, shows that the particle size distribution evolves rapidly from bimodal to monomodal distributions being displaced towards higher particle sizes values. This confirms that large and stable flocs are formed. In the case of BHMW, the evolution of $d_{\mathrm{p} 10}$ with flocculation time leads to the same conclusions withdrawn from the analysis of $d_{\mathrm{p} 50}$, as we can see in Fig. 5 .

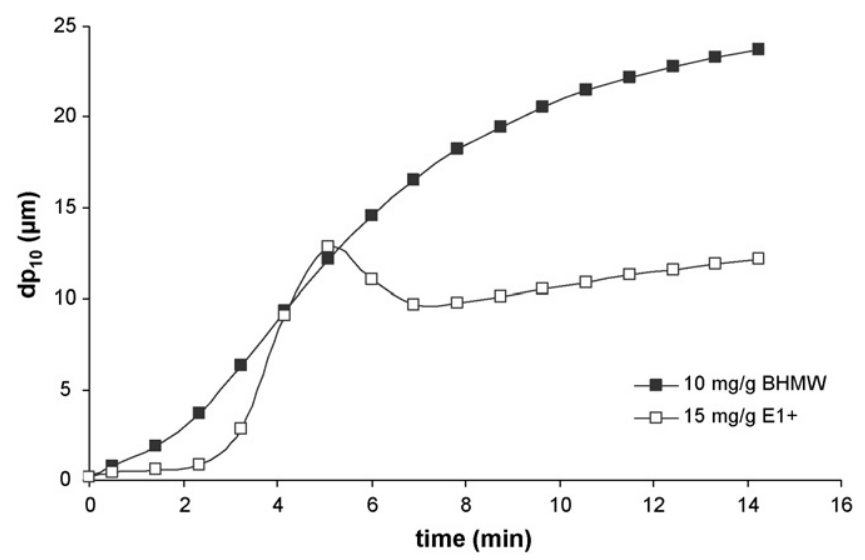

Fig. 5. $d_{\mathrm{p} 10}$ evolution as a function of flocculation time for optimum flocculant dosage.

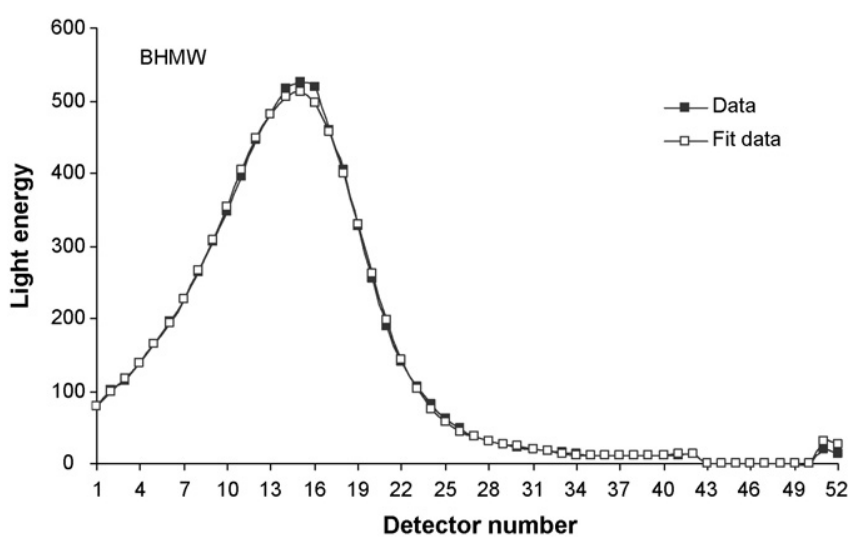

Fig. 6. Data and fitting curve based on Mie theory at the end of flocculation for $10 \mathrm{mg} / \mathrm{g}$ of BHMW.

The flocs size distribution was determined using the Mie theory and a very good agreement of the fitting of data with the optical model was found, as can be seen in Fig. 6. This agreement confirms the accuracy of the LDS technique and of the scattering model used.

Assuming that the flocs size at the end of flocculation is the parameter that defines the optimum flocculant dosage, Fig. 7 shows that for BHMW the maximum floc size was obtained with a flocculant concentration of $10 \mathrm{mg} / \mathrm{g}$, as already anticipated by the preliminary optimum dosage tests. On the contrary, for E1+, the maximum floc size was obtained for $15 \mathrm{mg} / \mathrm{g}$ (Figs. 2 and 7) whereas the previous dosage tests have indicated as optimum concentration $9 \mathrm{mg} / \mathrm{g}$ (Fig. 1). It is, nevertheless, reasonable that the optimum flocculant dosage is smaller for BHMW since, due its tendency to adopt a flatter conformation on the particle surface, the same polymer chain fills a larger area of the free particle surface and thus the saturation point is reached with a lower polymer quantity. Fig. 8 shows, as well, that only for $15 \mathrm{mg} / \mathrm{g}$ of E1+ or higher concentrations the particle size distribution of the flocs reaches a monomodal configuration.

The flocculation kinetics and the floc stability are the characteristics that most distinguish the two flocculants. In fact, with $\mathrm{E} 1+$, the floc size reaches a maximum, followed by a slight

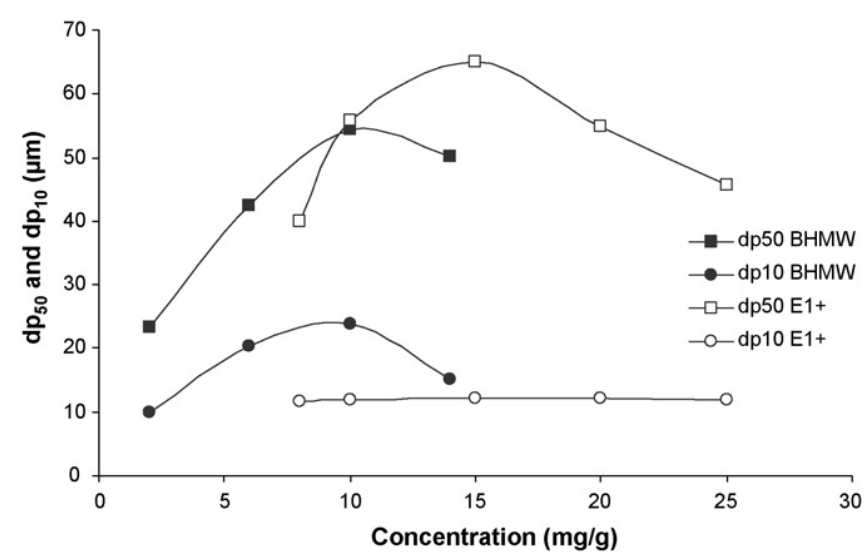

Fig. 7. $d_{\mathrm{p} 10}$ and $d_{\mathrm{p} 50}$ floc sizes at the end of the flocculation process as a function of flocculant concentration for both C-PAM flocculant. 


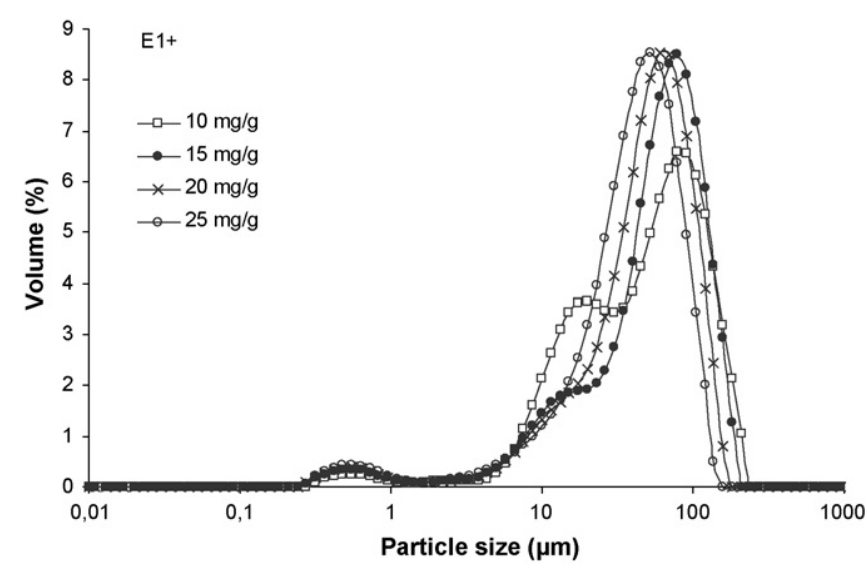

Fig. 8. Flocs size distribution at the end of flocculation process for several E1+ concentrations.

decrease until a final situation of stability is achieved (Fig. 2). This is a consequence of the predominance of the bridging mechanism during the first stage, when conformation is not so important as aggregation and the polymer adsorbed at the particle surface produces bonds with a completely extended configuration [1]. After the maximum floc size, conformation dominates and the flocs tend to become more compact, thus with a smaller mean size.

Comparing now the flocs size distributions at different times after the polymer addition $(10 \mathrm{mg} / \mathrm{g})$ during the polymer conformation stage, the amount of the small flocs increases with time (Fig. 9). These results suggest that floc size decreases after reaching the maximum size (Fig. 2) both as a result of the breakage of the flocs by the hydrodynamic forces and of the change of the conformation of the adsorbed polymer. For this flocculant, the $10 \mathrm{mg} / \mathrm{g}$ dosage is low (below the optimum dosage identified previously) and, therefore, coarser flocs with fewer bonds per floc are formed, resulting in flocs that are too weak and susceptible to break down due to the shear forces.

This agrees with the evolution of the $d_{\mathrm{p} 10}$ with time (Fig. 5). When E1+ is studied, it can be seen that $d_{\mathrm{p} 10}$ decreases after the maximum flocs size but then increases again, during the conformation stage. Smaller flocs that resulted from the break up of the larger ones, continue to flocculate during this period

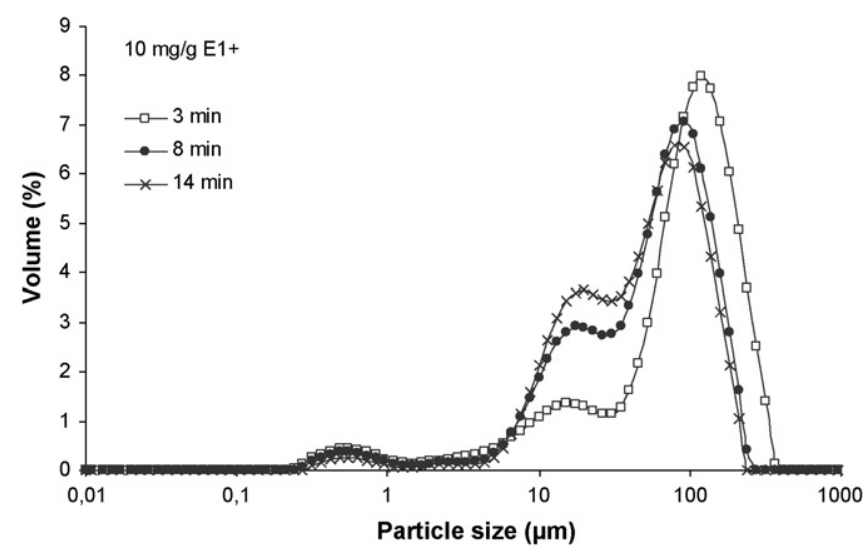

Fig. 9. Flocs size distribution during conformation phase for $10 \mathrm{mg} / \mathrm{g} \mathrm{E} 1+$.
Table 1

Median floc size at the end of flocculation process calculated for optimal flocculant dosage (comparison between LDS and optical microscopy)

\begin{tabular}{lll}
\hline Flocculant & $d_{\mathrm{p} 50}$ by LDS $(\mu \mathrm{m})$ & $d_{\mathrm{p} 50}$ by image analysis $(\mu \mathrm{m})$ \\
\hline BHMW & 55 & 54 \\
E1+ & 65 & 61 \\
\hline
\end{tabular}

and tend to behave in a different way than the coarser flocs. This phenomenon is again more pronounced as the flocculant concentration increases, due to the existence of an excess of polymer available in the suspension. These results demonstrate the relevance of monitoring the complete particle size distribution to detect the end of the flocculation process or, at least, the interest of associating information on both $d_{\mathrm{p} 50}$ and $d_{\mathrm{p} 10}$ for that purpose. In fact, for $\mathrm{E} 1+$, the study of the $d_{\mathrm{p} 50}$ diameter evolution alone has proven to be insufficient to define the end of the flocculation process.

From Table 1 it may be concluded that LDS and optical microscopy with image analysis, although being different techniques, give similar results for the median size of the flocs. However, it should be emphasized that, besides being laborious and time consuming image analysis considers just a few particles (between 100 and a 120 for the samples analysed) and, thus, the sample is always less representative than the one tested in LDS.

\subsection{Obscuration control}

As mentioned before, the quality of the signal obtained by LDS is closely related to the obscuration parameter. As flocs grow, the number of scattering entities decreases and obscuration decreases as well (Fig. 10). Besides, in the beginning of the flocculating process, when the rate of flocs growth is higher, obscuration decreases rapidly, whereas in the end, when the rate of flocs growth is small and flocs reach a steady state, obscuration tends to stabilize. For both polymers, obscuration is always higher than 5\% until the end of flocculation, and so the validity of the results is guaranteed.

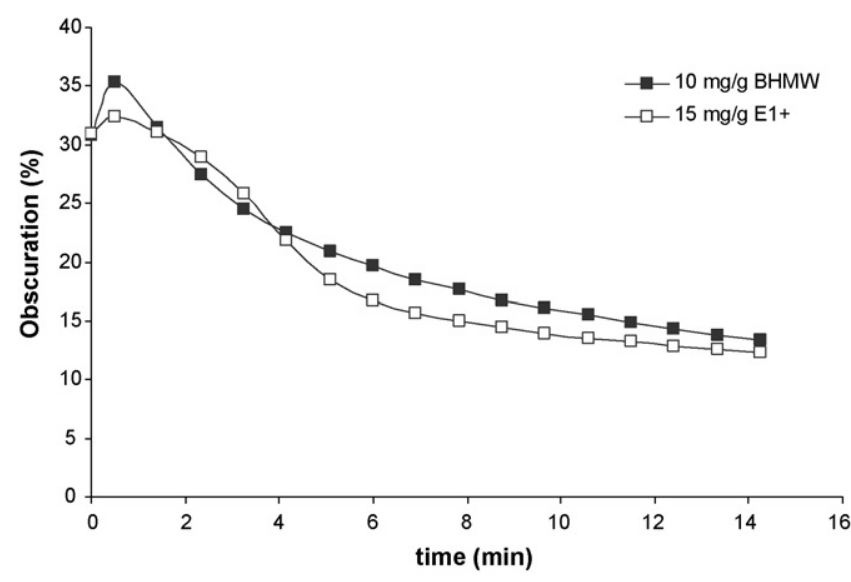

Fig. 10. Obscuration as a function of flocculation time for BHMW concentrations. 


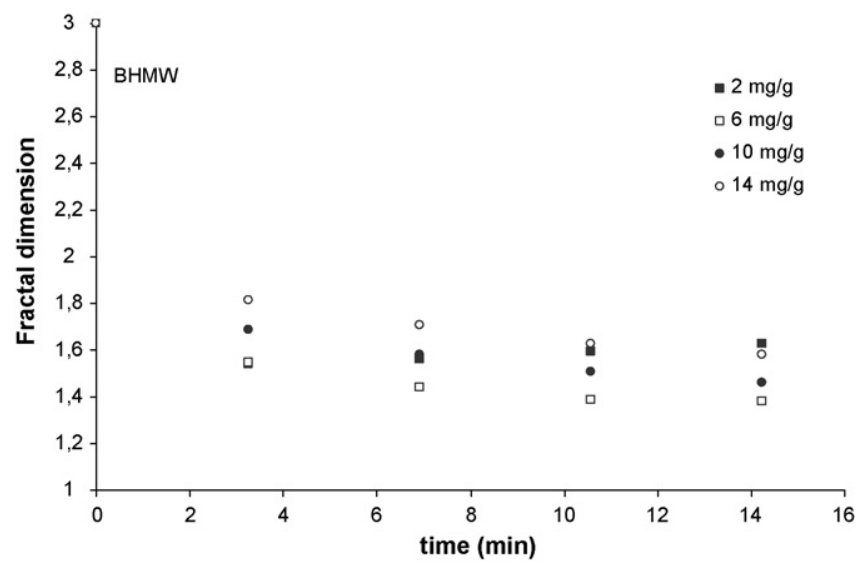

Fig. 11. Mass fractal dimension during flocculation process for several BHMW concentrations.

\subsection{Mass fractal dimension}

Figs. 11 and 12 show the mass fractal dimension $\left(d_{\mathrm{F}}\right)$ variation during the flocculation process induced by BHMW and E1+, respectively, and Table 2 summarizes the corresponding mass fractal dimensions at the end of the flocculation process, for all the concentrations studied.

For both flocculants, the mass fractal dimension decreases abruptly during the first minutes of flocculation, when a rapid growth of the floc median size occurs. In fact, as the flocs grow, porosity increases and the number of primary particles occupying a fixed volume of the aggregate decreases, resulting in a decrease in the mass fractal dimension [24]. This decrease is higher during the first minutes of flocculation because then the solid single PCC particles (high mass fractal dimension) are turning into particle aggregates, as a consequence of the polymer bonds. In any case, by looking at the scattering intensity pattern it can be said that only primary aggregation is obtained [4] and not secondary aggregation (aggregation of smaller aggregates), probably due to the fact that flocculation is being conducted in a turbulent environment. The fitting of the scattering intensity data is very good and thus, the Rayleigh-Gans-Debye theory can be used to extract the mass fractal dimension $[4,21]$.

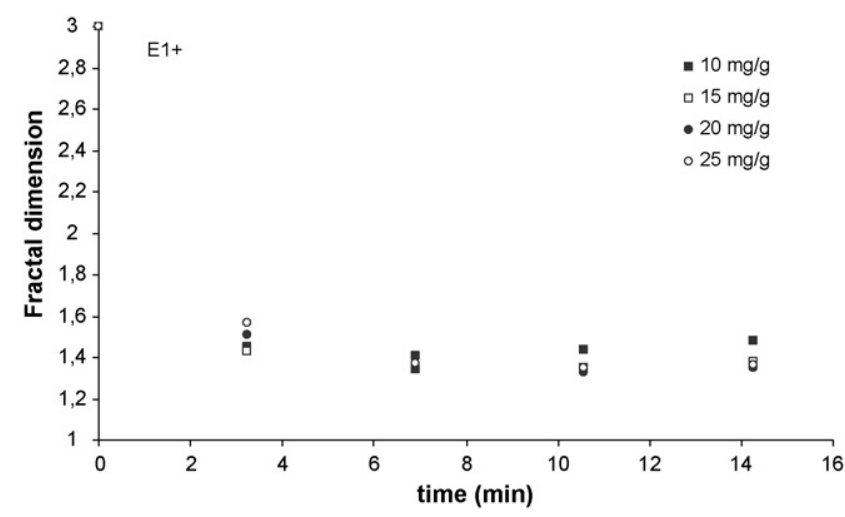

Fig. 12. Mass fractal dimension during flocculation process for several E1+ concentrations.
For BHMW, $d_{\mathrm{F}}$ decreases until stabilization whereas for $\mathrm{E} 1+, d_{\mathrm{F}}$ decreases and then increases slightly. Additionally, for BHMW, the mass fractal dimension increases as the flocculant concentration increases over $6 \mathrm{mg} / \mathrm{g}$. This is in agreement with the fact that the patching mechanism is more pronounced for higher flocculant concentrations, thus producing denser flocs.

For E1+, the final value of $d_{\mathrm{F}}$ does not vary notably with flocculant concentration because bridging is the single mechanism that takes place, whereas for BHMW there are two competing mechanisms dependent on the flocculant concentration. However, during the first minutes of flocculation induced by E1+, $d_{\mathrm{F}}$ increases slightly with the flocculant concentration, as expected considering the flocculation behaviour described before: when concentration decreases, the flocculation rate is faster and the degree of polymer conformation is lower, increasing the distance between the particles and thus producing more porous flocs. The slight increase of $d_{\mathrm{F}}$ during the final phase of the flocculation process is a consequence of the compaction of the flocs by polymer flattening. The highest value of $d_{\mathrm{F}}$ at the end of the flocculation process occurs for the lower polymer concentration $(10 \mathrm{mg} / \mathrm{g})$, since these flocs break down more easily resulting thereafter in denser aggregates.

Comparing now the mass fractal dimension of the two flocculants (Table 2), it is possible to observe that, for the optimal concentration, BHMW produces denser flocs than E1+, confirming that the flocs obtained by the patching mechanism, usually associated with higher polymer charge density, are denser than those obtained by the bridging mechanism.

\subsection{Zeta potential measurements}

Fig. 13 shows the results of the zeta potential measurements. The optimum dosage of BHMW, $10 \mathrm{mg} / \mathrm{g}$, corresponds to a value of the zeta potential of $25 \mathrm{mV}$, whereas the optimum dosage of $\mathrm{E} 1+, 15 \mathrm{mg} / \mathrm{g}$, corresponds to a value of the zeta potential of $15 \mathrm{mV}$. Therefore, since the flocculation process occurs for values of zeta potential far from the isoelectrical point $(0 \mathrm{mV})$, it may be concluded that flocculation is not occurring by the charge neutralization mechanism.

Due to the cationic character of the polyelectrolytes, the PCC particles, initially negatively charged $(-27 \mathrm{mV})$, become positively charged with the addition of flocculant. The larger zeta potential values obtained with BMHW are a result of its higher charge density. The reduction of the zeta potential during the flocculation process is an indication of polymer conformation

Table 2

Mass fractal dimension at the end of flocculation process for the two flocculants

\begin{tabular}{lll}
\hline Concentration $(\mathrm{mg} / \mathrm{g})$ & BHMW & E1+ \\
\hline 2 & 1.63 & - \\
6 & 1.38 & - \\
10 & 1.46 & 1.48 \\
14 & 1.58 & - \\
15 & - & 1.38 \\
20 & - & 1.35 \\
25 & - & 1.36
\end{tabular}




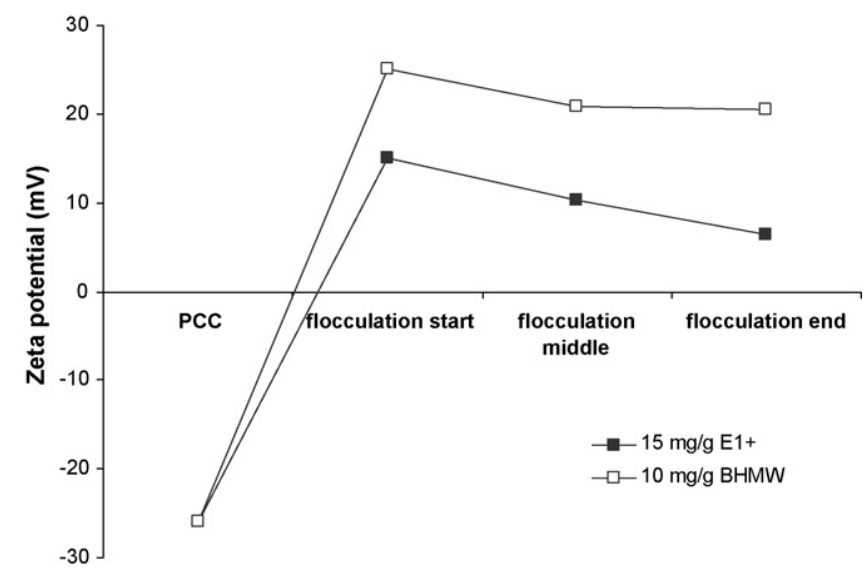

Fig. 13. Zeta potential at different phases of flocculation process for the optimum dosage.

$[20,22,30]$. However, for both flocculants, this parameter cannot be used to determine the optimum flocculant dosage since it does not allow assessing neither the mechanism nor the kinetics of the flocculation process.

\subsection{Flocculation kinetics}

Flocculation kinetics was evaluated by the evolution of the median floc size with flocculation time. Eq. (1) stands for the beginning of the process, when aggregation dominates and the floc size increases:

$d_{\mathrm{p}_{50}}=A \mathrm{e}^{k_{1} t}$

where $A$ is a pre-exponential factor $(\mu \mathrm{m}), t$ is the time ( $\mathrm{min})$ and $k_{1}$ is the kinetic constant $\left(\min ^{-1}\right)$, which depends on the polymer concentration.

When the aggregation starts to decrease as flocculation proceeds, two-distinct kinetics can be identified depending on the type of polymer. For BHMW, the experimental data can be adjusted to Eq. (2), identical to Eq. (1) but where the values of the kinetic constant $k_{2}\left(\mathrm{~min}^{-1}\right)$ are much smaller because the flocs growth rate decreases significantly and the flocs size tends to stabilize:

$d_{\mathrm{p}_{50}}=A \mathrm{e}^{k_{2} t}$

As for E1+, the evolution of the median flocs size with time is better described by Eq. (3) since, as seen before, the size of the flocs decreases after reaching a maximum, due to several factors, like polymer conformation and compaction or break-down:

$d_{\mathrm{P}_{50}}=A \mathrm{e}^{1 / k_{2} t}$

This Equation also stands for very low concentrations of BHMW since, again, as aggregation was very fast, conformation occurred only after the maximum floc size was reached.

Figs. 14 and 15 show again the evolution of the median floc sizes with time (as in Figs. 2 and 3) and the fitting lines obtained with the kinetics equations (for BHMW at $2 \mathrm{mg} / \mathrm{g}$, Eq. (3) was used for the second part of the curve). The corresponding kinetics constants $k_{1}$ and $k_{2}$, for the distinct concentrations of each floccu-

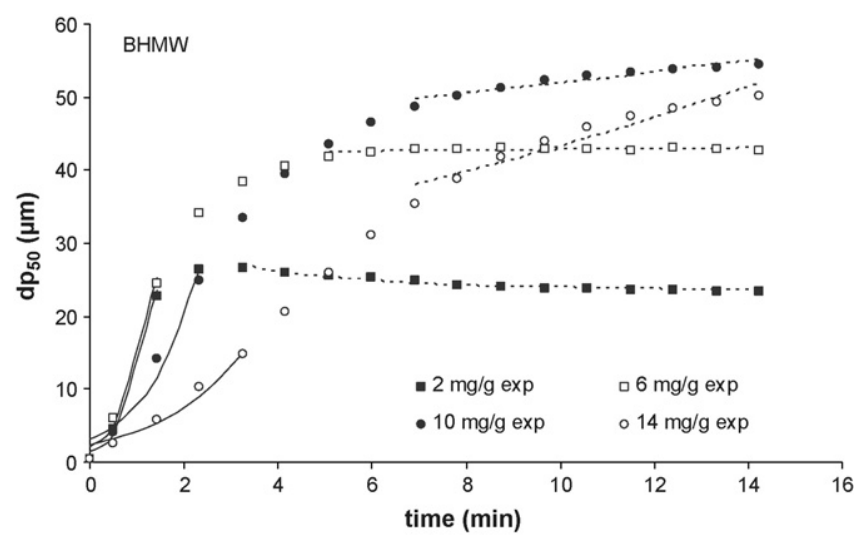

Fig. 14. Evolution of measured values and fitting curves of $d_{\mathrm{p} 50}$ with BHMW

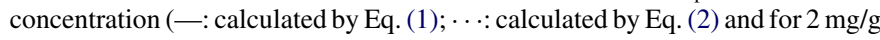
by Eq. (3)).

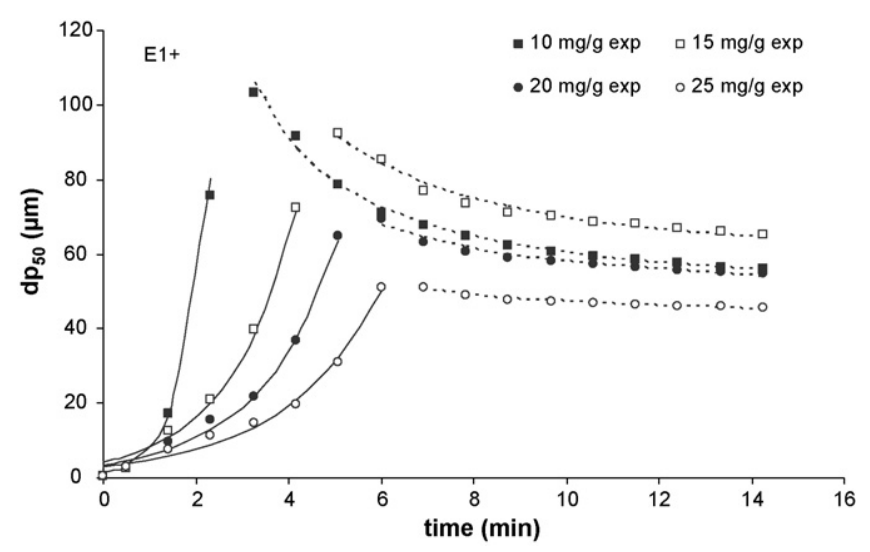

Fig. 15. Evolution of measured values and fitting curves of $d_{\mathrm{p} 50}$ with E1+ concentration (—: calculated by Eq. (1); $\cdots$ : calculated by Eq. (3)).

lant, are plotted in Figs. 16 and 17, respectively for BHMW and $\mathrm{E} 1+$. It must be stressed that for both polymers, there is always a transition zone, more pronounced in the case of BHMW, which is not accounted for in the proposed empirical modelling strategy. In fact, the approach followed is based on the thesis that during the flocculation process there are two distinct kinetic behaviours as a result of the predominance of different mechanisms in each

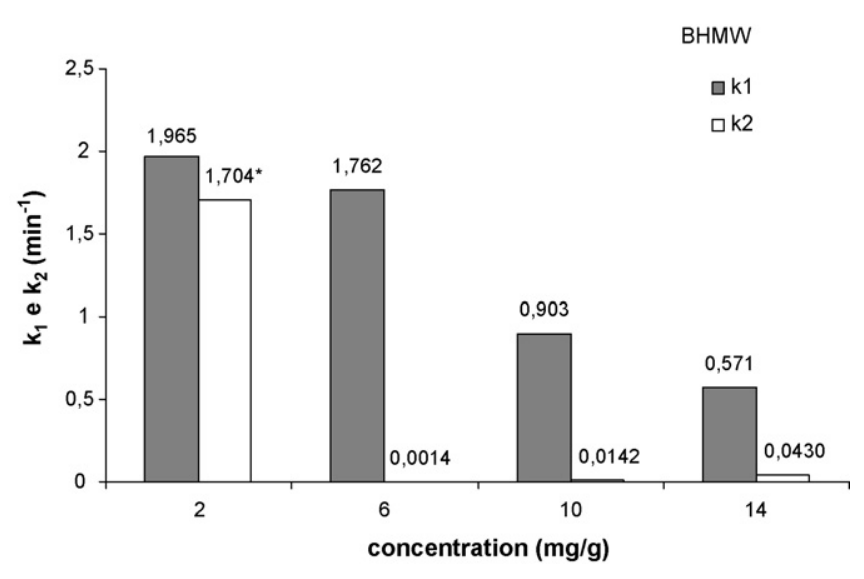

Fig. 16. Kinetics constants of the two flocculation regions for BHMW (*: adjusted with Eq. (3)). 


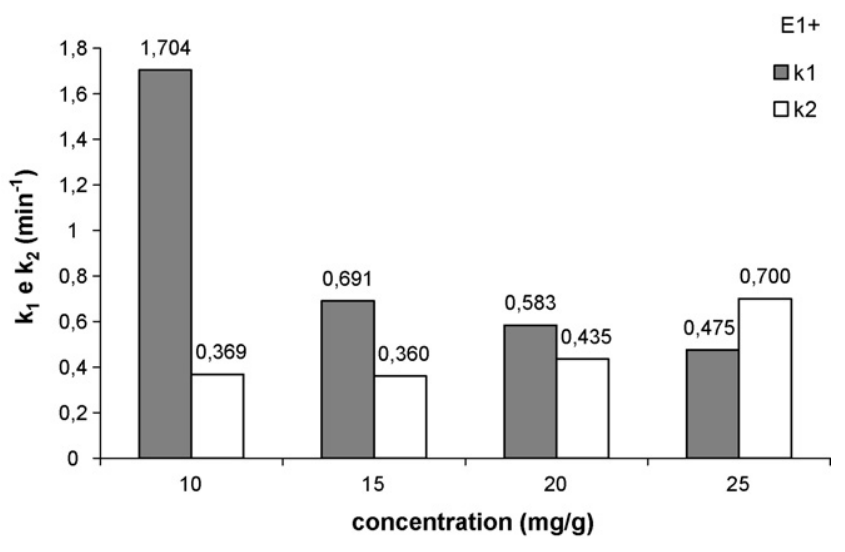

Fig. 17. Kinetics constants of the two flocculation regions for E1+.

zone. In the first zone (first minutes of the flocculation process), aggregation predominates; in the second zone (after reaching the maximum floc size), reconformation of the polymer or floc breakage predominate.

From Figs. 14 and 15 it can be seen that the fitting of the proposed models to the experimental data is very good. In the case of E1+ it is easy to define the two regions for the flocculation process and the transition from the first to the second region is very sharp. For BHMW, on the contrary, it is more difficult to separate the two regions because aggregation and break-up can occur simultaneously, mainly at the middle of the flocculation process. In this case, the transition region is smooth and, due to this, Eq. (1) was only adjusted to the first points of the flocculation process, whereas Eqs. (2) or (3) were used for the last points.

For BHMW, $k_{2}$ is related to the stability of the flocs at the end of the flocculation process. However, since, at the end of the aggregation stage, the flocs were already very stable, $k_{2}$ is low. The flocs stabilization rate decreases as polymer concentration increases, meaning that at higher concentrations the flocs stabilization is delayed by the aggregation process that has not yet finished. For $\mathrm{E} 1+, k_{2}$ represents predominantly the rate of polymer chains conformation after the aggregation step and high values of $k_{2}$ correspond to a higher degree of polymer conformation at the end of that stage (indeed, as discussed before, the flocs size stabilizes more rapidly as the flocculant concentration increases and this is represented by a high value of $k_{2}$ ). For this polymer the increase in the values of $k_{2}$ with flocculant concentration is in agreement with the fact that the flocs stabilization rate also increases. Because of a slower aggregation, flocs at the end of the aggregation phase have a configuration already closer to the final one, and so stabilization was faster.

The kinetic constant $k_{1}$ decreases as the flocculant concentration increases due to the fact that aggregation takes place more slowly because at dosages higher than the optimal to cover $50 \%$ of the particle surface, the possible orientations of particle to efficiently collide decreases. Since the values of $k_{1}$ are, in general, larger for BHMW than for E1+, it may be concluded that aggregation induced by BHMW is faster than the one produced by $\mathrm{E} 1+$, as a consequence of its higher charge density.
In general, for the two polymers, a comparison between $k_{1}$ and $k_{2}$ values shows that the aggregation process is faster than the stabilization process. As the flocculant concentration increases flocculation becomes limited by the aggregation process, which is slower, and the stabilization process becomes less important.

This study shows that the kinetic model depends strongly on the type of polymer and different empirical equations have to be considered for distinct polymers. In the present study, the charge density of the polymer is the parameter that influences most the flocculation process. Due to the different polymer charge density, different mechanisms were involved in the flocculation process and, therefore, the kinetic model reflected those mechanisms. Nevertheless, since no general theory is yet available to describe flocculation by polymeric additives [2] these empirical models are of high importance, for instance to the design of process equipment where flocculation takes place. In the case of systems involving the flow of flocculated suspensions (pipes, mixing tanks, headboxes in papermaking, etc.) flocculation kinetics have to be considered in the flow model or in the population balance model. Thus, to be able to obtain experimentally, practical information on the kinetics of those processes is of utmost importance and that can be easily obtained by using the LDS technique.

\section{Conclusions}

The results obtained in this study demonstrated the advantage of using the LDS technique to evaluate and understand the flocculation process and to determine the flocs characteristics. The developed experimental methodology allows, in a single integrated test, the acquisition of information on the evolution with time of flocs dimensions and structure and also the evaluation of flocs resistance and flocculation kinetics. This led to the definition of the optimum flocculant dosage and to the understanding of the flocculation mechanisms involved that could be correlated with the mass fractal dimension of the flocs. With this method, it was possible to study the influence of polyelectrolyte charge density and polymer concentration on the flocculation process of PCC, used in papermaking, and on flocs properties.

Thus, it is legitimate to conclude that LDS is a valuable tool to assess the performance of polymeric flocculants, being particularly suited to study flocculation in a turbulent environment.

The results obtained by image analysis confirmed the validity of the LDS results. Furthermore, it was demonstrated that the LDS technique is a better tool to determine flocs characteristics than other traditional techniques like image analysis or hindered settling.

The comparison of two polymers with high molecular weight shows that for medium charge density the flocculation process occurs by bridging mechanism while when the charge density is very high the patching mechanism becomes important and occurs simultaneously with bridging. As the flocculant concentration increases the flocculation rate decreases. The flocculation concentration also affects the flocs size stabilization rate.

The zeta potential measurements indicate the existence of polymer conformation during the flocculation process but they 
did not allow obtaining information about the optimal dosage, the flocculation mechanism or the flocculation kinetics.

It was possible to quantify the initial flocculation kinetics, the values obtained for the kinetic constants agreeing with the conclusions mentioned previously and having proved that charge density affects strongly the flocculation process. This information can be most important, for instance when modelling the flow of suspensions undergoing flocculation in process equipment.

\section{Acknowledgements}

The authors thank the European Project Nodeszeloss for financial support and AQUA+TECH Specialties SA (La Plaine, Geneva, Switzerland) for supplying the flocculant samples.

\section{References}

[1] J. Gregory, The action of polymeric flocculants, flocculation, in: Proceedings of Sedimentation and Consolidation, Engineering Foundation Conference, Georgia, USA, 1985, pp. 125-137.

[2] Ad.A. Berlin, V.N. Kislenko, Kinetic model of suspension flocculation by polymers, Colloid Surf. 104 (1995) 67-72.

[3] Ad.A. Berlin, I.M. Solomentseva, V.N. Kislenko, Suspension flocculation by polyelectrolytes: experimental verification of a developed mathematical model, J. Colloid Interf. Sci. 191 (1997) 273-276.

[4] S. Biggs, M. Habgood, G.J. Jameson, Yao-de Yan, Aggregate structures formed via a bridging flocculation mechanism, Chem. Eng. J. 80 (2000) $13-22$.

[5] T.N. Prabhu, R. Jagannadha, K. Prashantha, Studies on flocculation of clay suspension by polyacrilamide, e-Polymers (2004) 57.

[6] A.R. Heath, A.B. Parisa, P.D. Fawell, J.B. Farrow, Polymer flocculation of calcite: relating the aggregate size to the settling rate, AIChE J. 52 (6) (2006) 1987-1993.

[7] A. Blanco, C. Negro, A. Hooimeijer, J. Tijero, Polymer optimization in paper mills by means of a particle size analyser: an alternative to zeta potential measurements, Appita J. 49 (1996) 113-116.

[8] A. Blanco, E. Fuente, C. Negro, J. Tijero, Flocculation monitoring: focused beam reflectance measurement as a measurement tool, Can. J. Chem. Eng. 80 (4) (2002) 734-740.

[9] K.E. Bremmell, G.J. Jameson, S. Biggs, Kinetic polyelectrolyte adsorption at the solid/liquid interface interaction forces and stability, Colloid Surf. 139 (1998) 199-211.

[10] A. Blanco, C. Negro, E. Fuente, J. Tijero, Effect of shearing forces and flocculant overdose on filler flocculation mechanisms and floc properties, Ind. Eng. Chem. Res. 44 (24) (2005) 9105-9112.
[11] A. Swerin, G. Risinger, L. Ödberg, Flocculation in suspensions of microcrystalline cellulose by microparticle retention aid systems, J. Pulp. Pap. Sci. 23 (8) (1997) 374-381.

[12] T. Tadros, Applied Surfactants: Principles and Applications, Wiley-VCH, Weinheim, 2005.

[13] T.G.M. Van de Ven, B. Alince, Heteroflocculation by asymmetric polymer bridging, J. Colloid Interf. Sci. 181 (1996) 73-78.

[14] D.N. Thomas, S.J. Judd, N. Fawcett, Flocculation modelling: a review, Water Res. 33 (1999) 1579-1592.

[15] P.T. Spicer, S. Pratsinis, J. Raper, R. Amal, G. Bushell, G. Meesters, Effect of shear schedule on particle size, density, and structure during flocculation in stirred tanks, Pow. Tech. 97 (1998) 26-34.

[16] M.A. Yukselen, J. Gregory, The reversibility of floc breakage, Int. J. Miner Process 73 (2004) 251-259.

[17] C. Negro, E. Fuente, A. Blanco, J. Tijero, Flocculation mechanism induced by phenolic resin/PEO and floc properties, AIChE J. 51 (3) (2005) 1022-1031.

[18] P.M. Claesson, E. Poptoshev, E. Blomberg, A. Dedinaite, Polyelectrolytemediated surface interactions, Adv. Colloid Interf. Sci. 114-115 (2005) 173-187.

[19] Z. Yan, Y. Deng, Cationic microparticle based flocculation and retention systems, Chem. Eng. J. 80 (2000) 31-36.

[20] T. Miyanishi, Effects of zeta potential on flocculation measurement in microparticle systems, Tappi J. 78 (11) (1995) 135-141.

[21] J.Y.H. Liao, C. Selomulya, G. Bushell, G. Bickert, R. Amal, On different approaches to estimate the mass fractal dimension of coal aggregates, Part. Part. Syst. Charact. 22 (2005) 299-309.

[22] M.G. Rasteiro, F.A.P. Garcia, M. del Mar Peréz, Applying LDS to monitor flocculation in papermaking, Part. Sci. Technol. 25 (2007), in press.

[23] G. Bushell, Forward light scattering to characterise structure of flocs composed of large particles, Chem. Eng. Sci. 111 (2006) 145-149.

[24] R.K. Chakraborti, K. Gardner, J. Atkinson, J. Van Benschoten, Changes in fractal dimension during aggregation, Water Res. 37 (2003) 873-883.

[25] M. Hermawan, T. Yang, G. Bushell, R. Amal, G. Bickert, A new approach in determining floc strength, Part. Syst. Anal., Harrogate, UK, 2003.

[26] ISO 13320-1 Particle Size Analysis-Laser Diffraction Methods, Part 1: General Principle, International Organization of Standardization, Genève, 1999.

[27] G.B.J. De Boer, C. de Weerd, D. Thoenes, H.W.J. Goossens, Laser diffraction spectroscopy: Fraunhofer diffraction versus Mie scattering, Part. Charact. 4 (1987) 14-19.

[28] J. Teixeira, Small-angle scattering by fractal systems, J. Appl. Cryst. 21 (1988) 781-785.

[29] M. Norell, J. Kjell, M. Persson, Retention and Drainage, Papermaking and Science Technology, Book 4: Papermaking Chemistry, L. Neimo, Finland, 1999, pp. 42-61.

[30] M.A. Hubbe, Charge-related measurements: a reappraisal. Part 2. Fibre-pad streaming potential, Paper Technol. 45 (8) (2004) 27-34. 